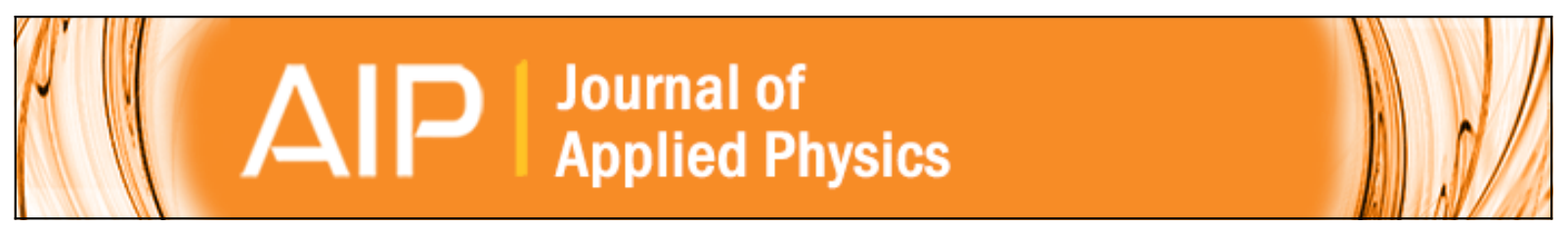

\title{
Theoretical model for geometry-dependent magnetoelectric effect in magnetostrictive/piezoelectric composites
}

Yaojin Wang, Davresh Hasanyan, Menghui Li, Junqi Gao, Jiefang Li, D. Viehland, and Haosu Luo

Citation: Journal of Applied Physics 111, 124513 (2012); doi: 10.1063/1.4729832

View online: http://dx.doi.org/10.1063/1.4729832

View Table of Contents: http://scitation.aip.org/content/aip/journal/jap/111/12?ver=pdfcov

Published by the AIP Publishing

\section{Articles you may be interested in}

Magnetoelectric coupling in sol-gel synthesized dilute magnetostrictive-piezoelectric composite thin films

J. Appl. Phys. 110, 036101 (2011); 10.1063/1.3610795

Present status of theoretical modeling the magnetoelectric effect in magnetostrictive-piezoelectric nanostructures. Part II: Magnetic and magnetoacoustic resonance ranges

J. Appl. Phys. 107, 053905 (2010); 10.1063/1.3313920

Present status of theoretical modeling the magnetoelectric effect in magnetostrictive-piezoelectric nanostructures. Part I: Low frequency and electromechanical resonance ranges

J. Appl. Phys. 107, 053904 (2010); 10.1063/1.3313919

A strong magnetoelectric voltage gain effect in magnetostrictive-piezoelectric composite Appl. Phys. Lett. 85, 3534 (2004); 10.1063/1.1786631

Theory of low-frequency magnetoelectric effects in ferromagnetic-ferroelectric layered composites J. Appl. Phys. 92, 7681 (2002); 10.1063/1.1522834

MIT LINCOLN

LABORATORY CAREERS

Discover the satisfaction of innovation and service

to the nation
- Space Control

- Air \& Missile Defense

- Communications Systems \& Cyber Security

- Intelligence, Surveillance and

Reconnaissance Systems

- Advanced
Electronics
- Tactical Systems
- Homeland
Protection
- Air Traffic Control

LINCOLN LABORATORY

MassachusetTs Institute OF TeChNOLOGY

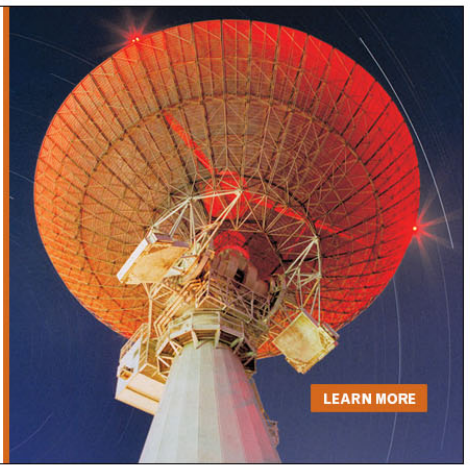




\title{
Theoretical model for geometry-dependent magnetoelectric effect in magnetostrictive/piezoelectric composites
}

\author{
Yaojin Wang, ${ }^{1, a)}$ Davresh Hasanyan, ${ }^{1}$ Menghui Li, ${ }^{1}$ Junqi Gao, ${ }^{1}$ Jiefang Li, ${ }^{1}$ D. Viehland, ${ }^{1}$ \\ and Haosu Luo ${ }^{2}$ \\ ${ }^{1}$ Materials Science and Engineering, Virginia Tech, Blacksburg, Virginia 24061, USA \\ ${ }^{2}$ Shanghai Institute of Ceramics, Chinese Academy of Sciences, 215 Chengbei Road, Jiading, Shanghai \\ 201800, China
}

(Received 13 February 2012; accepted 19 May 2012; published online 28 June 2012)

\begin{abstract}
A quasistatic theoretical model including geometry effect is presented for predicting the magnetoelectric (ME) coefficients in a ME multilayer composite consisting of magnetostrictive and piezoelectric layers. The model is developed based on average-field method considering the geometry effect. The model characterizes the ME coefficient in terms of not only the parameters of two composite components and the thickness fraction but also the length and width fractions for the piezoelectric or magnetostrictive components. Analytical predictions indicate that the width and length fractions strongly influence the maximum ME coefficient and the corresponding thickness fraction also. Clearly, geometry effects cannot be ignored in predicting ME coefficient. Theoretical ME coefficients are also compared to experimental test data, demonstrating excellent agreement. (C) 2012 American Institute of Physics. [http://dx.doi.org/10.1063/1.4729832]
\end{abstract}

\section{INTRODUCTION}

The magnetoelectric (ME) effect is potentially useful for magnetic field sensors, electric-write magnetic-read memory devices, current measurement probes for high-power electric transmission systems, and energy harvesting. ${ }^{1,2}$ Since the intrinsic ME response of single-phase ME materials is too low for device applications, intensive experimental and theoretical studies have focused on the extrinsic ME coupling in multiphase composites combining magnetostrictive and piezoelectric components. ${ }^{3}$ The extrinsic ME effect in composites is a product-property, i.e., mechanical deformation in the magnetostrictive phase results in a dielectric polarization in the piezoelectric phase. ${ }^{3,4}$ Experimentally, various ME composites in different systems have been investigated in recent years, ${ }^{4}$ including (i) particulate bulk ceramic ME composites of piezoelectric ceramics and ferrites; (ii) two-phase ME composites of magnetostrictive alloy and piezoelectric ceramics/-or single crystals; ${ }^{5,6}$ (iii) three-phase ME composites; ${ }^{7,8}$ and (iv) nanostructured thin-films of ferroelectric and magnetic oxides. ${ }^{9}$

A milestone in the development of ME composites was the appearance of ones operated in multi-push-pull mode, consisting of magnetostrictive Metglas alloys and piezoelectric $\mathrm{Pb}\left(\mathrm{Mg}_{1 / 3} \mathrm{Nb}_{2 / 3}\right) \mathrm{O}_{3}-\mathrm{PbTiO}_{3}$ (PMN-PT) or $\mathrm{Pb}(\mathrm{Zr}, \mathrm{Ti}) \mathrm{O}_{3}$ (PZT) fibers with interdigitated-electrodes. ${ }^{5,6} \mathrm{~A}$ giant $\mathrm{ME}$ coefficient $\alpha_{\mathrm{E}}$ of $52 \mathrm{~V} / \mathrm{cm}$. Oe and an extremely low equivalent magnetic noise of $5.1 \mathrm{pT} / \sqrt{ } \mathrm{Hz}$ have been observed in a Metglas/PMN-PT sensor at low frequencies. ${ }^{6}$ Theoretically, several methods have been employed to model the ME coupling at both low frequency and at resonance, including (i) averagefield, ${ }^{10,11}$ (ii) Green function, ${ }^{4,12}$ and (iii) equivalent circuit. $^{4,13}$ The ME theoretical models have implicated that the 2-2 connectivity in laminate composites results in the highest ME coupling amongst the various composite systems: ${ }^{14}$

${ }^{a)}$ Electronic mail: yaojin@vt.edu. please note that predicted ME coefficients had a similar trends as the experimental data. However, there has still remained some notable differences in the overall magnitude of the ME coefficient, such as the theoretical predictions were several times higher than the experimental values and/or the theoretical results had no dependence on composite geometry, whereas the experimental values were sensitive thereof. Clearly, a theoretical analysis of the geometry-dependent ME coefficient is crucial for a complete understanding of ME composites.

In this paper, an analytical model for ME coupling has been developed based on an average-field method by considering the geometry effect. The predicted ME coefficient depended not only on the parameters of the components but also on the composite geometry. The analytical study has been compared to experimental measurements with good agreement. The most significant outcome of our model is that it can predict the ME coefficient of multilayer composites consisting of magnetostrictive and piezoelectric phases having different geometries.

\section{THEORETICAL MODELING DEVELOPMENT}

We consider a multilayer structure composite consisting of $N$-layer piezoelectric and $(N+1)$-layer magnetostrictive materials, as shown in Fig. 1(a). While the derivation is specific to this sandwich-like laminate, the approach is valid for any multilayer magnetostrictive/piezoelectric ME composite. For a poled piezoelectric phase of symmetry $\infty m$ (i.e., transverse isotropy), the constitutive equations can be written for the strain and electric displacement as

$$
{ }^{p} S_{i}={ }^{p} S_{i j}^{p} T_{j}+{ }^{p} d_{k i}^{p} E_{k}, \quad{ }^{p} D_{k}={ }^{p} d_{k i}^{p} T_{i}+{ }^{p} \varepsilon_{k n}{ }^{p} E_{n},
$$

where ${ }^{p} S_{i}$ and ${ }^{p} T_{j}$ are the strain and stress tensor components of the piezoelectric phase; ${ }^{p} E_{k},{ }^{p} E_{n}$, and ${ }^{p} D_{k}$ are the vector 


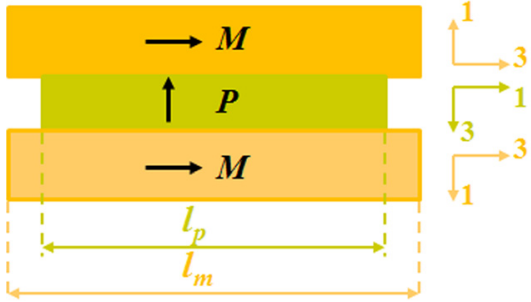

(a)

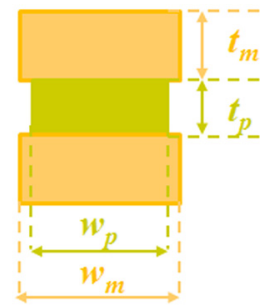

(b)
FIG. 1. Schematic diagram and geometry of a multilayer magnetostrictive/ piezoelectric composite: (a) width direction and (b) length direction. The arrows $M$ and $P$ designate the magnetization and polarization directions, respectively.

components of the electric field and electric displacement; ${ }^{p} s_{i j}$ and ${ }^{p} d_{k i}$ are the compliance and piezoelectric coefficients; and ${ }^{p} \varepsilon_{k n}$ is the permittivity matrix of the piezoelectric phase.

The magnetostrictive phase is assumed to have cubic symmetry (i.e., isotropy), and thus the strain and magnetic induction can be described by the equations

$$
{ }^{m} S_{i}={ }^{m} s_{i j}{ }^{m} T_{j}+{ }^{m} q_{k i}{ }^{m} H_{k}, \quad{ }^{m} B_{k}={ }^{m} q_{k i}{ }^{m} T_{i}+{ }^{m} \mu_{k n}{ }^{m} H_{n},
$$

where ${ }^{m} S_{i}$ and ${ }^{m} T_{j}$ are strain and stress tensor components of the piezoelectric phase; ${ }^{m} H_{k},{ }^{m} H_{n}$, and ${ }^{m} B_{k}$ are the vector components of the magnetic field and magnetic flux induction; ${ }^{m} s_{i j}$ and ${ }^{m} q_{k i}$ are compliance and piezomagnetic coefficients; and ${ }^{m} \mu_{k n}$ is the permeability matrix of the magnetostrictive phase.

The analysis assumes small deformations, linear materials coefficients, and perfect interfacial bonding. For the solutions of Eqs. (1) and (2), the following boundary conditions were used:

$$
\begin{aligned}
& { }^{m} S_{2}={ }^{p} S_{2}, \quad{ }^{m} S_{3}={ }^{p} S_{1}, \quad{ }^{m} T_{1}={ }^{p} T_{3}=0, \\
& { }^{m} T_{3}(1-\nu)(1-\omega)+{ }^{p} T_{1} \nu \omega=0, \\
& { }^{m} T_{2}(1-\nu)(1-\tau)+{ }^{p} T_{2} \nu \tau=0, \quad{ }^{p} D_{3}=0,
\end{aligned}
$$

where $\quad v=t_{p} /\left(t_{p}+2 t_{m}\right), \quad \omega=w_{p} /\left(w_{p}+w_{m}\right), \quad$ and $\tau=l_{p} /\left(l_{p}+l_{m}\right) ; w_{\mathrm{m}}, t_{\mathrm{m}}$, and $l_{\mathrm{m}}$ denote the width, thickness, and length of magnetostrictive; and $w_{\mathrm{p}}, t_{\mathrm{p}}$, and $l_{\mathrm{p}}$ denote the width, thickness, and of piezoelectric layer. Please note that for a $N$-layer piezoelectric/ $(N+1)$-layer magnetostrictive multilayer ME composite, the thickness fraction for piezoelectric component should be given as $v=N t_{p} /\left[N t_{p}+(N+1) t_{m}\right]$.

From Eqs. (1), (2), and (3), we can obtain

$$
\begin{gathered}
{\left[{ }^{m} s_{12}+\frac{{ }^{p} s_{12} v \xi}{(1-v)(1-\xi)}\right]{ }^{m} T_{2}+\left[{ }^{m} s_{11}+\frac{{ }^{p} s_{11} v \omega}{(1-v)(1-\omega)}\right]{ }^{m} T_{3}-{ }^{p} d_{31}{ }^{p} E_{3}=-{ }^{m} q_{33}{ }^{m} H_{3},} \\
{\left[{ }^{m} s_{11}+\frac{{ }^{p} s_{11} v \xi}{(1-v)(1-\xi)}\right]{ }^{m} T_{2}+\left[{ }^{m} s_{12}+\frac{{ }^{p} s_{12} v \omega}{(1-v)(1-\omega)}\right]^{m} T_{3}-{ }^{p} d_{31}{ }^{p} E_{3}=-{ }^{m} q_{31}{ }^{m} H_{3},} \\
\frac{{ }^{p} d_{31} v \xi}{(1-v)(1-\xi)}{ }^{m} T_{2}+\frac{{ }^{p} d_{31} v \omega}{(1-v)(1-\omega)}{ }^{m} T_{3}-{ }^{p} \varepsilon_{33}{ }^{p} E_{3}=0 .
\end{gathered}
$$

Next, we define the effective compliance coefficients as

$$
\begin{aligned}
A & =\left[{ }^{m} s_{12}+\frac{{ }^{p} s_{12} v \xi}{(1-v)(1-\xi)}\right], \\
B & =\left[{ }^{m} s_{11}+\frac{{ }^{p} s_{11} v \omega}{(1-v)(1-\omega)}\right], \\
C & =\left[{ }^{m} s_{11}+\frac{{ }^{p} s_{11} v \xi}{(1-v)(1-\xi)}\right], \\
D & =\left[\frac{{ }^{m} s_{12}+{ }^{p} s_{12} v \omega}{(1-v)(1-\omega)}\right],
\end{aligned}
$$

and the effective piezoelectric coefficient as

$$
X=\frac{{ }^{p} d_{31} v \xi}{(1-v)(1-\xi)}, \quad Y=\frac{{ }^{p} d_{31} v \omega}{(1-v)(1-\omega)}
$$

From Eq. (4) and the above definitions, the ME coefficient in terms of the electric field $\left(E_{3}=v^{p} E_{3}\right.$ (Ref. 14)) response to an applied magnetic field $\left(H_{3}\right)$ can be expressed as

$$
\begin{aligned}
\alpha_{E}= & -\left({ }^{m} q_{33} v\right) \times \frac{(Y C-X D)}{-B X^{p} d_{31}+A Y^{p} d_{31}+B C^{p} \varepsilon_{33}-Y C^{p} d_{31}-A D^{p} \varepsilon_{33}+X D^{p} d_{31}} \\
& -\left({ }^{m} q_{31} v\right) \times \frac{B X-A Y}{-B X^{p} d_{31}+A Y^{p} d_{31}+B C^{p} \varepsilon_{33}-Y C^{p} d_{31}-A D^{p} \varepsilon_{33}+X D^{p} d_{31}} .
\end{aligned}
$$

Chang and Carman ${ }^{15}$ obtained an expression for the L-T mode ME coefficient of the form 
TABLE I. Material parameters for CFO, PZT, $\mathrm{Fe}_{74.4} \mathrm{Co}_{21.6} \mathrm{Si}_{0.5} \mathrm{~B}_{3.3} \mathrm{Mn}_{0.1} \mathrm{C}_{0.1}$ (Metglas), and PMN-PT used for theoretical modeling.

\begin{tabular}{lccccc}
\hline \hline Materials & ${ }^{m} s_{11}$ or ${ }^{p} s_{11}\left(10^{-12} \mathrm{~m}^{2} / \mathrm{N}\right)$ & ${ }^{m} s_{12}$ or ${ }^{p} s_{12}\left(10^{-12} \mathrm{~m}^{2} / \mathrm{N}\right)$ & ${ }^{m} q_{12}\left(10^{-9} \mathrm{~m} / \mathrm{A}\right)$ & ${ }^{m} q_{11}\left(10^{-9} \mathrm{~m} / \mathrm{A}\right)$ & ${ }^{p} d_{31}\left(10^{-12} \mathrm{C} / \mathrm{N}\right)$ \\
\hline CFO $^{\mathrm{a}}$ & 6.5 & -2.4 & & -1.88 & 0.556 \\
Metglas $^{\mathrm{b}}$ & 10 & -5.2 & -21.3 & 50.3 & $\ldots$ \\
PZT $^{\mathrm{a}}$ & 15.3 & -5 & $\cdots$ & $\ldots$ & -175 \\
PMN-PT $^{\mathrm{c}}$ & 57.3 & -34.1 & $\cdots$ & $\ldots$ & 1750 \\
\hline
\end{tabular}

${ }^{\mathrm{a}}$ Cited from Ref. 14

${ }^{\mathrm{b}}$ Cited from Ref. 16

${ }^{\mathrm{c}}$ Measured.

$\alpha_{E, L-T}=\frac{-{ }^{p} d_{31}(1-v) v\left({ }^{m} q_{31}+{ }^{m} q_{33}\right)}{p_{\left.\varepsilon_{33}\left[\left({ }^{m} s_{11}+{ }^{m} s_{12}\right)(1-v)+\left({ }^{p} s_{11}+{ }^{p} s_{12}\right) v\right)\right]-2\left({ }^{p} d_{31}\right)^{2} v}}$.

The above equation corresponds to a special case of our geometry-dependent $\mathrm{ME}$ theory in which one assumes $w_{\mathrm{p}}=w_{\mathrm{m}}$ and $l_{\mathrm{p}}=l_{\mathrm{m}}$ (i.e., $\xi=0.5$ and $\omega=0.5$ ). Thus, for the special case, the effective coefficients have the relationships of $A=D, B=C$, and $X=Y$.

The theoretical model that we obtained allows for a detailed analysis of the ME effect in the magnetostrictive/ piezoelectric composite structure. Analysis can be done on the effects not only for the material parameters of the two phases but also for the geometries of the two components. Variations in thickness and width of either piezoelectric or magnetostrictive components can be analyzed. Based upon such analysis, we predicted the general trends of the ME coefficient and compared the modeling results to other theoretical and experimental values. The material characteristics for theoretical modeling are listed in Table I.

\section{VALIDATION OF THE THEORETICAL MODEL}

In this section, the theoretical model is validated, via comparisons to other modeling results and to experimental data. Based on the model, analysis can be done on the effects of the parameter of the composite components and on variation in geometry of the components. We will then compare our predictions to other theoretical models and observe the general trends of the ME coefficient using the same materials with property parameters given in Table I. We analyzed the theoretical models for two different ME composites with cobalt ferrite (CFO) or Metglas as the magnetostrictive components, and PZT or PMN-PT as the piezoelectric ones.

First, we discuss a system of primary interest in earlier investigations: CFO/PZT multilayers. ${ }^{14}$ Since the previous models assumed the same lengths and widths for the magnetostrictive and piezoelectric layers, we estimated the $\mathrm{ME}$ coefficient as a function of the thickness fraction $\nu$ for the piezoelectric phase under the case of a length fraction of $\xi=0.5$ and width fraction of $\omega=0.5$. The results in Fig. 2(a) were obtained assuming a length fraction of $\xi=0.5$ (i.e., $l_{\mathrm{p}}=l_{\mathrm{m}}$ ) for piezoelectric phase in the CFO-PZT multilayer using the material parameters given in Table I. Obviously, a ME coupling was absent in the individual phase $(v=0$, CFO; $v=1$, PZT). For various width fraction $\omega$, as $v$ was increased from $0<v<1$, the value of $\alpha_{\mathrm{E}}$ increased and reached a maximum value of $\alpha_{\mathrm{E}, \max }$ at $v_{\max }$. This maximum was due to an increased elastic interaction between piezoelectric and magnetostrictive layers. The value of $\alpha_{E}$ then decreased with a further increase of $v$. It can be seen that the $\alpha_{\mathrm{E}, \max }$ decreased as the width fraction $\omega$ increased and $v_{\max }$ shifted to CFO-rich phase fractions. In particular, the plot for $\omega=0.5$ was the same as the results based on earlier models. ${ }^{14}$ Figure 2(b) shows the $\omega$ dependence of $\alpha_{\mathrm{E}, \max }$ and correspondingly $v_{\max }$, plotted directly from Fig. 2(a). With increasing $\omega$, we find (i) a near-linear increase occurs in the maximum value of $\alpha_{E}$ and (ii) the ME coupling reaches a maximum at progressively lower values of $v_{\max }$. The ME coefficient was increased by a factor of $5.3 \times$ from
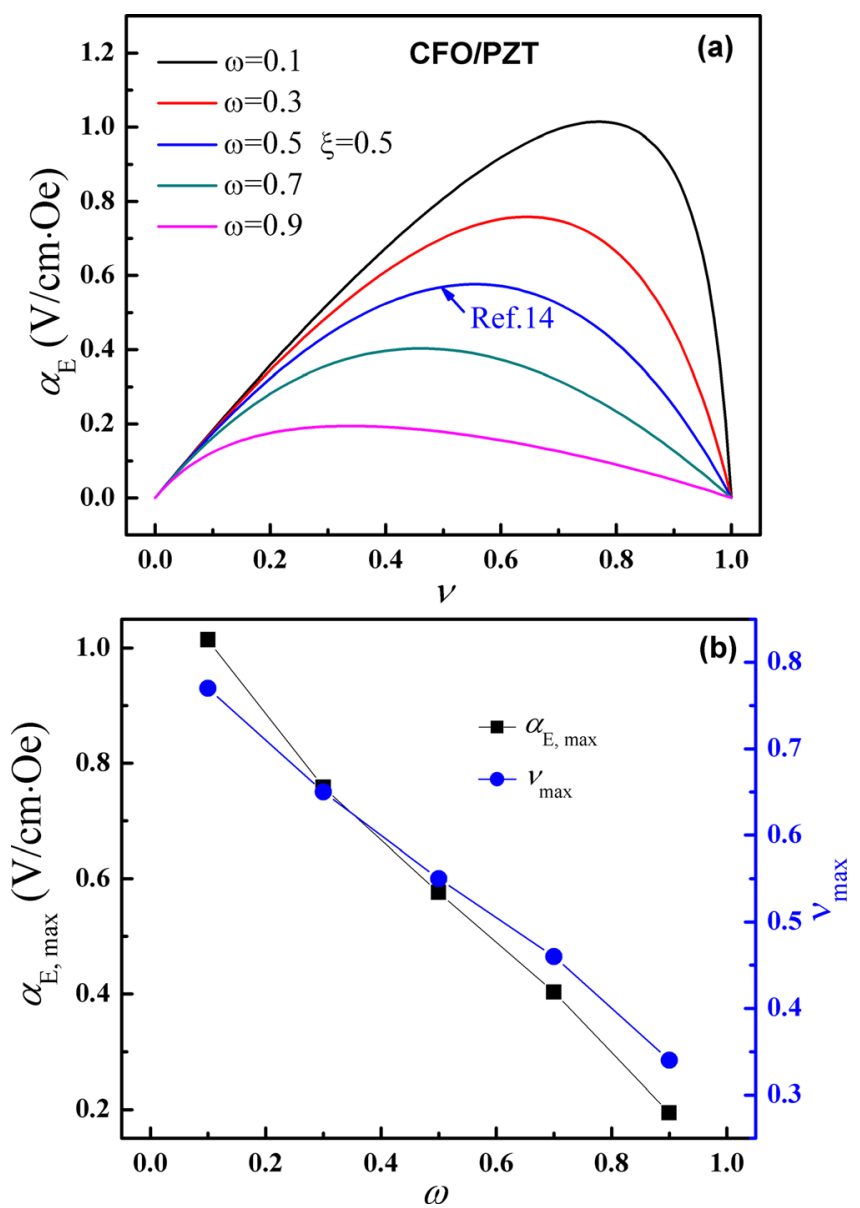

FIG. 2. (a) Predicted dependence of $\alpha_{\mathrm{E}}$ on thickness fraction $\nu$ for various width fractions $\omega$ and length fractions $\xi=0.5$ (i.e., $l_{\mathrm{p}}=l_{\mathrm{m}}$ ) for piezoelectric phase in the CFO/PZT multilayer composite. (b) Variation with $\omega$ of maximum $\alpha_{\mathrm{E} \text {,max }}$ and the corresponding $\nu_{\max }$ under $\xi=0.5$. 
$0.19 \mathrm{~V} / \mathrm{cm} \cdot$ Oe to $1.01 \mathrm{~V} / \mathrm{cm} \cdot$ Oe, when the width fraction $\omega$ was changed from 0.1 to 0.9 .

Then, we considered another special case for a width fraction of $\omega=0.5$ for CFO-PZT composites. The thickness fraction $\nu$ dependence of the ME coefficient $\alpha_{\mathrm{E}}$ for various length fraction $\xi$ is shown in Fig. 3(a). It can be seen that both $\alpha_{\mathrm{E} \text {,max }}$ and $v_{\max }$ were not sensitive to changes in the length fraction $\xi$, as the width fraction $\omega$ was altered. Figure 3(b) shows $\alpha_{\mathrm{E}, \max }$, and the corresponding $v_{\max }$, as a function of $\xi$. As a response to changes in width, the value of $\alpha_{\mathrm{E} \text {,max }}$ exhibited a near-linear decrease with increasing $\xi$. We can see that $\alpha_{\mathrm{E}}$ was increased by about $1.3 \times$ from $0.5 \mathrm{~V} / \mathrm{cm} \cdot \mathrm{Oe}$ to $0.64 \mathrm{~V} / \mathrm{cm} \cdot \mathrm{Oe}$, when $\xi$ was changed from 0.1 to 0.9 . From these changes in $\omega$ [Fig. 2(a)] and $\xi$ [Fig. 3(a)], it is important to note that enhancement in $\alpha_{\mathrm{E}}$ occurred more significantly by increasing the width of the magnetostrictive phase, than by increasing its length.

Another multilayer ME composite of importance is $\mathrm{Fe}_{74.4} \mathrm{Co}_{21.6} \mathrm{Si}_{0.5} \mathrm{~B}_{3.3} \mathrm{Mn}_{0.1} \mathrm{C}_{0.1}$ (Metglas) alloys and piezoelectric $0.7 \mathrm{~Pb}\left(\mathrm{Mg}_{1 / 3} \mathrm{Nb}_{2 / 3}\right) \mathrm{O}_{3}-0.3 \mathrm{PbTiO}_{3}$ (PMN-PT) single crystals. Due to their high piezomagnetic and piezoelectric coefficients, these composites possess the highest ME effects and highest sensitivity to small magnetic field variation. Using our model, we estimated $\alpha_{\mathrm{E}}$ for Metglas/PMN-PT of
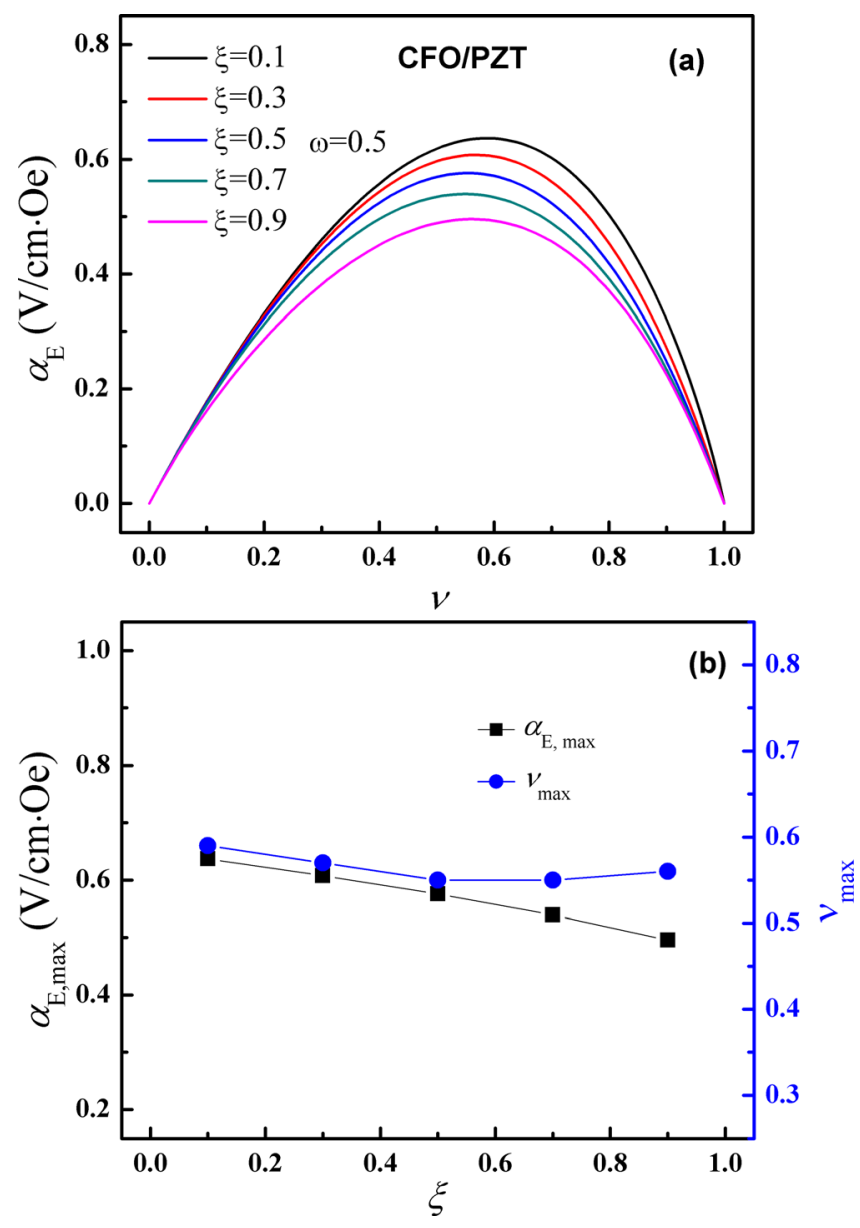

FIG. 3. (a) Predicted dependence of $\alpha_{\mathrm{E}}$ on thickness fraction $\nu$ under various length fractions $\xi$ and width fractions $\omega=0.5$ (i.e., $w_{\mathrm{p}}=w_{\mathrm{m}}$ ) for the piezoelectric phase in the CFO/PZT multilayer composite. (b) Variation with $\xi$ of maximum $\alpha_{\mathrm{E}, \max }$ and the corresponding $\nu_{\max }$ under $\omega=0.5$. various geometries, similar to that which was done for $\mathrm{CFO} /$ PZT. Representative results for $\xi=0.5$ are presented in Fig. 4(a). Similar trends with piezoelectric thickness fraction were observed for this composite. The maximum theoretical value of $\alpha_{\mathrm{E}}$ was as high as $23.9 \mathrm{~V} / \mathrm{cm}$. Oe for $\xi=0.5$ and $\omega=0.5$ under the constant of $\nu_{\max }=0.39$, which was a factor of $41.4 \times$ larger than that of the CFO/PZT composites. The significant enhancement in $\alpha_{\mathrm{E}}$ for Metglas/PMN-PT was due to the higher piezomagnetic coefficient for Metglas and the higher piezoelectric coefficient for PMN-PT. The variation in $\alpha_{\mathrm{E}, \max }$ and the corresponding $\nu_{\max }$ with $\omega$ is shown in Fig. 4(b). Both $\alpha_{\mathrm{E}, \max }$ and corresponding $\nu_{\max }$ dramatically decreased with increase of $\omega$.

Figure 5(a) shows $\alpha_{\mathrm{E}}$ as function of PMN-PT thickness fraction $\nu$ for various length fraction $\xi$ under a width fraction of $\omega=0.5$. The variation in $\alpha_{\mathrm{E}, \max }$ and $\nu_{\max }$ with $\xi$ is shown in Fig. 5(b), which was not as significant as that with $\omega$. An interesting observation is that the plot for $\xi=0.9$ was much flatter than the other composite geometries, with relatively higher values of $\alpha_{\mathrm{E}}$ maintained for $0.2<\nu<0.8$.

It is important to compare the theoretical predictions with experimental results. However, there have been few systematic studies of ME coefficient changes with component geometry. In case of experimental error due to sample fabrication,
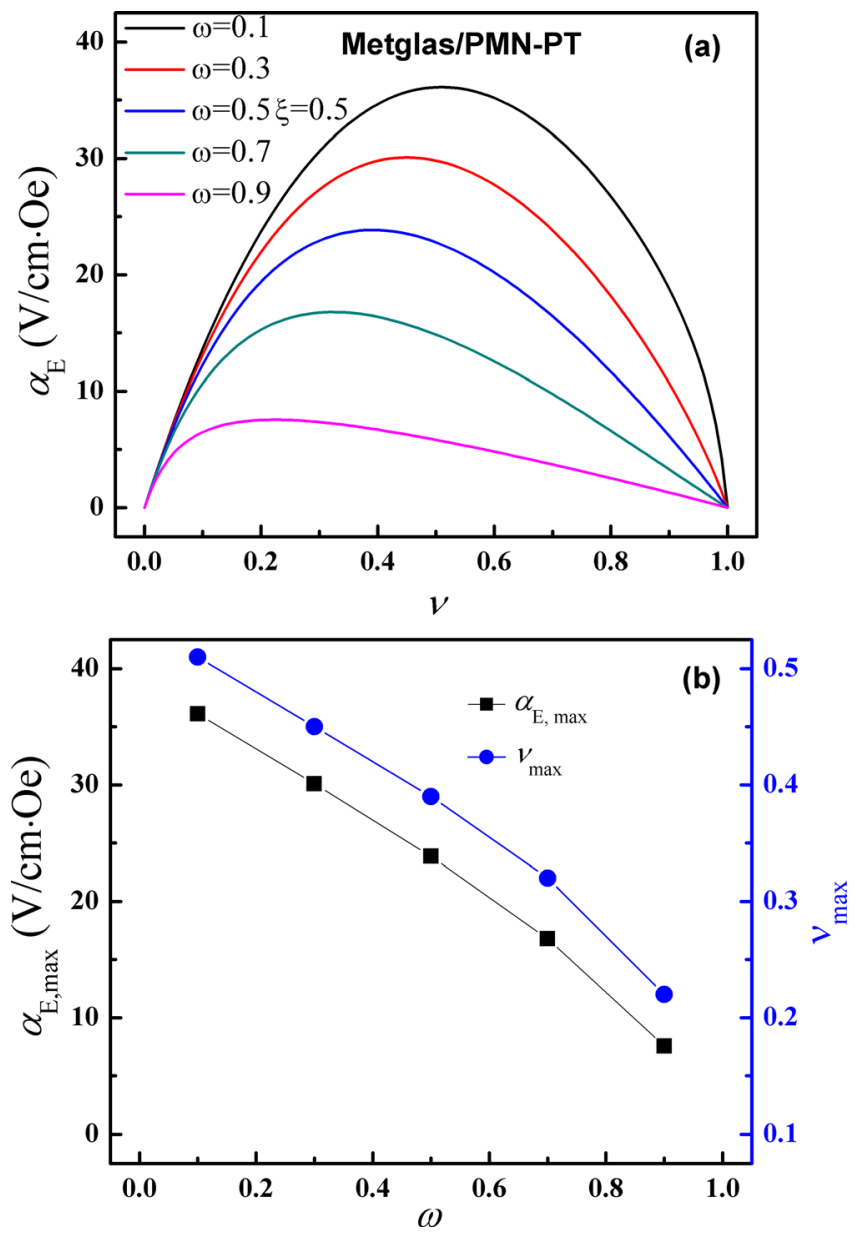

FIG. 4. (a) Predicted dependence of $\alpha_{\mathrm{E}}$ on thickness fraction $\nu$ under various width fractions $\omega$ and length fractions $\xi=0.5$ (i.e., $l_{\mathrm{p}}=l_{\mathrm{m}}$ ) for the piezoelectric phase in the Metglas/PMN-PT multilayer composite. (b) Variation with $\omega$ of maximum $\alpha_{\mathrm{E}, \max }$ and the corresponding $\nu_{\max }$ under $\xi=0.5$. 

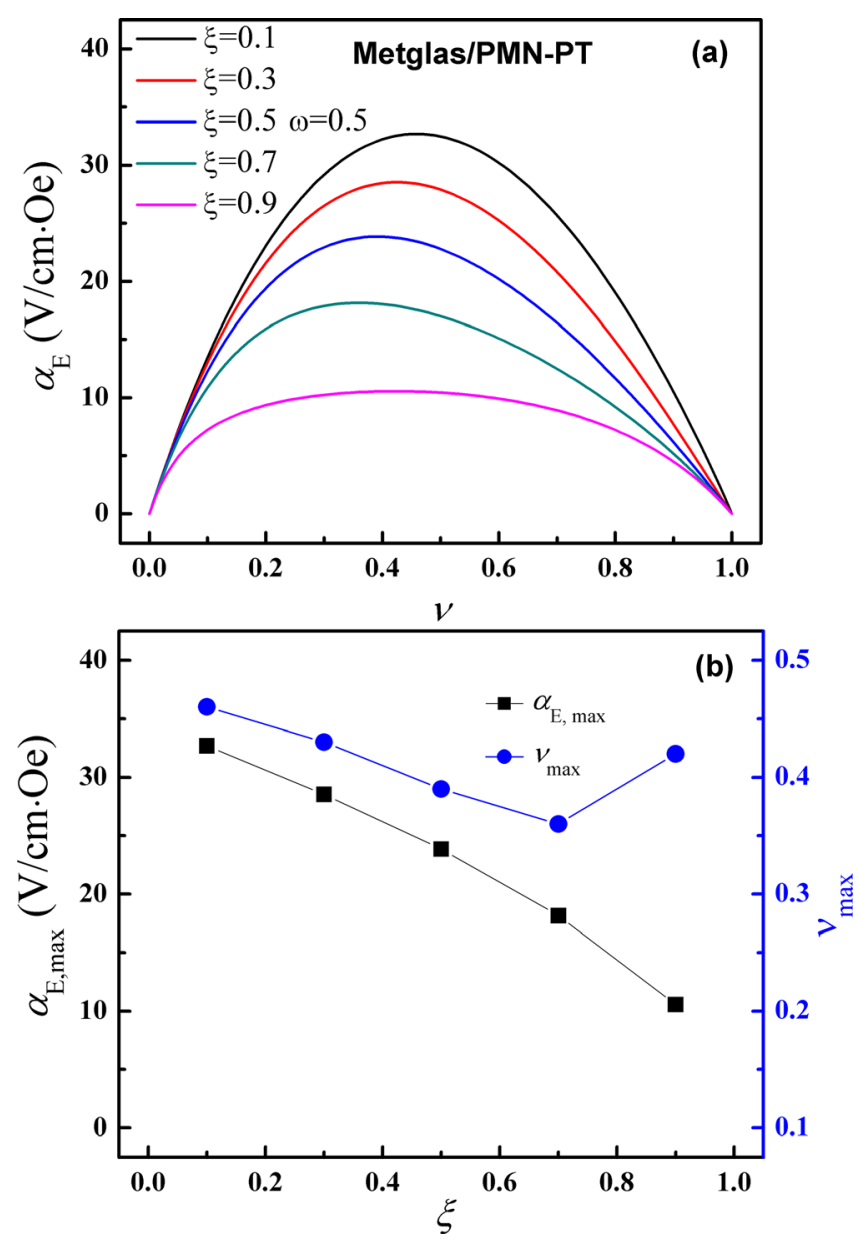

FIG. 5. (a) Predicted dependence of $\alpha_{\mathrm{E}}$ on thickness fraction $\nu$ under various length fractions $\xi$ and width fractions $\omega=0.5$ (i.e., $w_{\mathrm{p}}=w_{\mathrm{m}}$ ) for the piezoelectric phase in the Metglas/PMN-PT multilayer composite. (b) Variation with $\xi$ of maximum $\alpha_{\mathrm{E}, \max }$ and the corresponding $\nu_{\max }$ under $\omega=0.5$.

only one Metglas/PMN-PT composite was made, in which a thickness poled PMN-PT piezoelectric single crystal layer $\left(20 \times 6 \times 0.3 \mathrm{~mm}^{3}\right.$ with a $\langle 110\rangle$ direction along thickness $)$ was sandwiched between two-layer longitudinally magnetized Metglas foils $\left(80 \times 18 \times 0.025 \mathrm{~mm}^{3}\right)$. After sample fabrication, the width of the Metglas foil in this sample was successively trimmed to dimensions of $15 \mathrm{~mm}, 12 \mathrm{~mm}, 9 \mathrm{~mm}$, and $6 \mathrm{~mm}$. The ME coefficient was characterized as function of DC magnetic bias $H_{\mathrm{DC}}$ for each width of the Metglas layers. After testing the ME coefficient for the sample for a Metglas width of $w_{\mathrm{m}}=6 \mathrm{~mm}$, the length of Metglas was successively trimmed from $80 \mathrm{~mm}$ to $60 \mathrm{~mm}, 50 \mathrm{~mm}, 40 \mathrm{~mm}, 30 \mathrm{~mm}$, and $20 \mathrm{~mm}$. At each length of the Metglas foils, the ME coefficient was measured. For these studies of changes in width, the thickness and length fractions of PMN-PT were $\nu=0.86$ and $\xi=0.2$, where the width fraction $\omega$ was designed to changes by trimming from 0.25 to $0.29,0.33,0.4$, and 0.5 . Figure 6 (a) shows the theoretical and experimental values of $\alpha_{\mathrm{E}}$ as function of $\omega$ for these various geometry, where the values of $\alpha_{\mathrm{E}}$ correspond to the maximum ones in $\alpha_{\mathrm{E}} \mathrm{vs} H_{\mathrm{DC}}$. It can be seen that the trend of theoretical and experimental values of $\alpha_{\mathrm{E}}$ exhibit excellent agreement and that the prediction is about a factor of $1.5 \times$ larger than the experimental data. During these length change investigations, the values of $\nu=0.86$ and
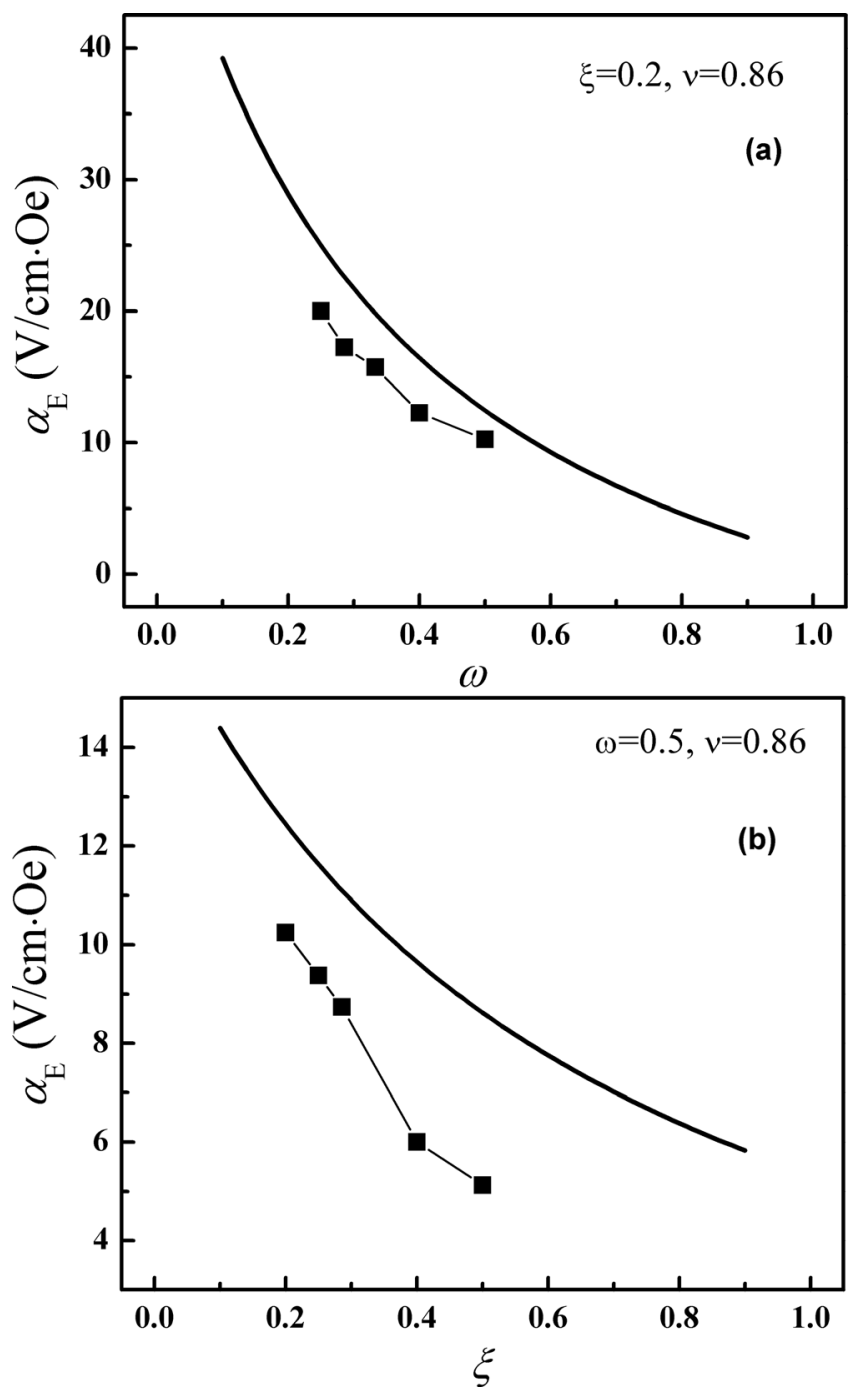

FIG. 6. (a) Comparison of theoretical (solid line) and experimental (line and symbol) $\alpha_{\mathrm{E}}$ as function of (a) width fraction $\omega$ under a length fraction $\xi=0.2$ and a thickness fraction $\nu=0.86$; (b) length fraction $\xi$ under a width fraction $\omega=0.5$ and a thickness fraction $\nu=0.86$.

$\omega=0.5$ were held constant, and the length fraction was designed to successively change with trimming from 0.2 to $0.25,0.29,0.4$, and 0.5 . In this case, the changes in the theoretical and experimental values of $\alpha_{\mathrm{E}}$ with $\xi$ are shown in Fig. 6(b). Similar trends for predicted and measured values can be seen.

\section{CONCLUSIONS}

A theoretical model including geometry effects has been developed for the longitudinal and transverse mode $\mathrm{ME}$ coefficients in magnetostrictive and piezoelectric laminate composites. The model employed length, width, and thickness fractions for the piezoelectric or the magnetostrictive layers to predict the geometry-dependent ME coefficient: rather than simply the volume fraction as given in previous models. The predictions indicate that the length, width, and thickness fractions significantly influence the ME coefficient, in addition to the parameters of the components. The model has been applied to multilayer composites of previous and 
present interests: i.e., CFO/PZT and Metglas/PMN-PT. The theoretical model predicts (i) the ME coefficient is enhanced as the width and the length fractions of the piezoelectric layer are decreased (i.e., wide and/or long magnetostrictive layer) under a constant thickness fraction; (ii) enhancements resulting from the width fraction are more significant than these from the length fraction; (iii) the width and length fractions strongly influence the optimal thickness fraction; and (iv) the ME coefficient of Metglas/PMN-PT multilayer composites are much higher than that in CFO/PZT composites for identical geometries. Analytical predictions for geometry-dependent ME coefficient were also compared to experimental data, and they are shown to be in an excellent agreement. Our results clearly demonstrate that a geometry effect must be incorporated for ME coefficient predictions in multilayer composite, especially for composite component of different widths and lengths.

\section{ACKNOWLEDGMENTS}

This work was sponsored by the Office of Naval Research.
${ }^{1}$ N. A. Spaldin and M. Fiebig, Science 309, 391 (2005).

${ }^{2}$ R. Ramesh and N. A. Spaldin, Nat. Mater. 6, 21 (2007).

${ }^{3}$ J. Ma, J. Hu, Z. Li, and C.-W. Nan, Adv. Mater. 23, 1062 (2011).

${ }^{4}$ C. W. Nan, M. I. Bichurin, S. X. Dong, D. Viehland, and G. Srinivasan, J. Appl. Phys. 103, 031101 (2008).

${ }^{5}$ S. X. Dong, J. Y. Zhai, J. F. Li, and D. Viehland, Appl. Phys. Lett. 89, 252904 (2006).

${ }^{6}$ Y. J. Wang, D. Gray, D. Berry, J. Q. Gao, M. H. Li, J. F. Li, and D. Viehland, Adv. Mater. 23, 4111 (2011).

${ }^{7}$ S. X. Dong, J. Y. Zhai, J. F. Li, and D. Viehland, J. Appl. Phys. 100, 124108 (2006).

${ }^{8}$ S.-C. Yang, C.-S. Park, K.-H. Cho, and S. Priya, J. Appl. Phys. 108, 093706 (2010).

${ }^{9}$ H. Zheng et al., Science 303, 661 (2004).

${ }^{10}$ M. I. Bichurin, V. M. Petrov, S. V. Averkin, and E. Liverts, J. Appl. Phys. 107, 053904 (2010).

${ }^{11}$ G. Srinivasan, E. T. Rasmussen, and R. Hayes, Phys. Rev. B 67, 014418 (2003).

${ }^{12}$ C.-W. Nan, G. Liu, Y. Lin, and H. Chen, Phys. Rev. Lett. 94, 197203 (2005).

${ }^{13}$ J. L. Dong and S. X. Dwight Viehland, IEEE Trans. Ultrason. Ferroelectr. Freq. Control 51, 794 (2004).

${ }^{14}$ M. Bichurin, V. Petrov, and G. Srinivasan, Phys. Rev. B 68, 054402 (2003).

${ }^{15}$ C.-M. Chang and G. Carman, Phys. Rev. B 76, 134116 (2007).

${ }^{16}$ Y. J. Wang, D. Hasanyan, M. H. Li, J. Q. Gao, J. F. Li, and D. Viehland, "Equivalent magnetic noise in multi-push-pull configuration magnetoelectric composites: model and experiment," IEEE Trans. Ultrason. Ferroelectr. Freq. Control (submitted). 